\title{
Modelling convection in A star atmospheres (Research Note)
}

\section{Bisectors and lineshapes of HD 108642}

\author{
Ch. Stütz \\ University of Vienna, Universitätsstrasse 7, 1010 Vienna, Austria \\ e-mail: christian.stuetz@univie.ac.at \\ Received 30 January 2009 / Accepted 3 July 2009 \\ ABSTRACT

\begin{abstract}
Context. We present a code, VeDyn, for modelling envelopes and atmospheres of A to $\mathrm{F}$ stars focusing on accurate treatment of convective processes.

Aims. VeDyn implements a highly sophisticated non-local convection model but is fast and can be used in practical astrophysical applications.

Methods. We developed the HME envelope solver for this convection model to consistently model the envelope together with the stellar atmosphere. The synthesis code SynthV was extended to account for the resulting velocity structure. Finally, we tested our approach on atomic line bisectors.

Results. It is shown that our synthetic line bisectors of HD 108642 bend towards the blue and are of a magnitude comparable to the observed ones.

Conclusions. Even though this approach of modelling convection requires the solution of a coupled system of nonlinear differential
\end{abstract} \\ equations, it is fast enough to be applicable to many of the investigation techniques relying on model atmospheres.
}

Key words. convection - stars: atmospheres - line: profiles - turbulence - stars: individual: HD 108642

\section{Introduction}

A physical mechanism most challenging to understand in $\mathrm{F}$ to early A stars is convection. It is one of the least understood phenomena taking place in these stars. Recently, several successful attempts to describe and to model convection in the envelope and the photosphere in more detail have been conducted. For instance, numerical simulations by Nordlund \& Stein (2000), Stein \& Nordlund (2003), Freytag \& Steffen (2004) and Kochukhov et al. (2007), and tests and applications of nonlocal convection models presented in Kupka (1999, HME), Kupka \& Muthsam (2007), and Kupka \& Montgomery (2002).

However, in practical applications like stellar spectrum analysis or stellar evolution modelling, the mixing length theory (Biermann 1948) is still most commonly used to model convection. Kupka (1996) and Heiter et al. (2002) implemented the local full spectrum turbulence model of Canuto and Mazzitelli $(1991,1992)$ and Canuto et al. (1996) in the widely used model atmosphere code AtLAs9 (Kurucz 1993). The complete Reynolds stress model (RSM) of Canuto and Dubovikov (1998, CD98) has been applied to envelopes of A-stars as well as white dwarfs (Kupka \& Montgomery 2002; Montgomery \& Kupka 2004). Their calculations are in qualitative agreement with 2D numerical simulations of Freytag (1995) and the resulting vertical velocites are consistent with observerved values for micro- and macroturbulence.

Extending this work, we investigated the possibility of applying the CD98 convection model also to stellar atmospheres. The various analysis methods of stellar spectra connected to or relying on stellar atmosphere modelling mostly use the micro- and macroturbulence parameters to account for those contributions to the lines shapes believed to originate from turbulent velocities. These, however, permit us only to model distortions symmetric with respect to the line center and are not inherent to the underlying stellar model.

In this publication, we show our extension of the HME envelope solver to model stellar envelopes together with the stellar atmosphere and a method to account for the derived velocity fields in spectrum synthesis. Following this approach, the structure and magnitude of the up- and downflows result from the model calculation and are then put into the derivation of spectral line profiles. Hence, the need for the parametrization of a microand marcroturbulence is expected to be considerably reduced.

We also present an application of our models to the star HD 108642 which has already been investigated by Landstreet (1998) and others concerning microturbulence, abundances and also bisectors.

\section{The models}

The convection model which we used is an extension of the CD98 approach, as described in Kupka and Montgomery (2002). The equations for the turbulent quantities are solved on a weighted mass grid. The weighting allows a non-equidistant spacing of the grid such that steep temperature gradients are resolved. All other quantities are derived on the same weighted mass grid in the envelope and on a $\log \left(\tau_{\text {ross }}\right)$ grid in the atmosphere. The transition region is determined automatically according to $\rho, P$ and $T$ at each iteration step. This way we could consistently model the stellar atmosphere together with 


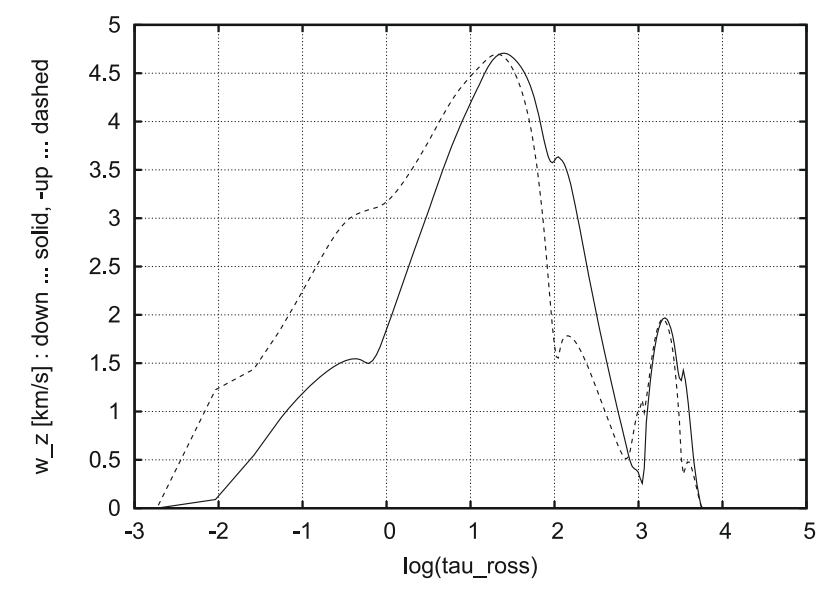

Fig. 1. Velocities $\left(-\omega_{\mathrm{z}}^{\text {up }}-\right.$ dashed, $\omega_{\mathrm{z}}^{\text {down }}-$ solid $)$ for HD 108642 derived from our model.

the envelope with feasible computational efforts. For the models presented in this paper we assumed spherical symmetry. We aim to use at least two sources for the opacities which are OPAL opacities (1996) in the envelope, and opacitiy distribution functions in the atmosphere. At present, however, we restrict ourselves to the OPAL routines, since they are faster and basic code development is still ongoing. Depending on the maximum size of time steps and the necessary relaxation time, one model requires 3 to $30 \mathrm{~h}$ to finish on a $2.3 \mathrm{GHz}$ single CPU machine.

\section{Synthesis and bisectors}

To derive synthetic spectra from our models and velocity profiles, we adopted the SynthV code of Vadim Tsymbal. Following the CD98 Eqs. (38d) and (50d) to (50f)

$$
\begin{aligned}
S_{w} & =\frac{\overline{\omega^{3}}}{\left.\overline{\left(\omega^{2}\right.}\right)^{3 / 2}} \\
\sigma & =0.5\left[1-S_{w}\left(4+S_{w}^{2}\right)^{-1 / 2}\right] \\
\omega_{\mathrm{z}}^{\text {up }} & =-\left(\frac{1-\sigma}{\sigma}\right)^{1 / 2} \bar{\omega}^{1 / 2} \\
\omega_{\mathrm{z}}^{\text {down }} & =\left(\frac{\sigma}{1-\sigma}\right)^{1 / 2} \bar{\omega}^{1 / 2}
\end{aligned}
$$

we can derive typical velocities $\omega_{\mathrm{z}}^{\text {up }}, \omega_{\mathrm{z}}^{\text {down }}$ and the filling factor $\sigma$ (fractional area occupied by the updrafts) from the mean vertical turbulent velocity $\overline{\omega^{2}}$ and the skewness $S_{w}$.

Figure 1 compares the absolute values of the up- and downdraft velocities we calculated. Note that at tau $=2 / 3$ $\left(\log \left(\tau_{\text {ross }}\right)=-0.176\right)$ these are in the range of typical values for the microturbulent velocity one would expect for an A type star. Contrary to CD98, our upstream velocities are negative, since in our frame of reference up means moving towards the observer.

The synthesis code SynthV of Tsymbal has been developed to allow to account for the upward and downward flow by means of separate radial velocity profiles. Their contributions to the total line profile are then weighted according to the filling factor (Fig. 2) and summed.

\section{HD 108642}

The Am star HD 10842 has been observed several times with an emphasis on its peculiar nature, abundance pattern and atmospheric velocity fields (Landstreet 1998). The observations

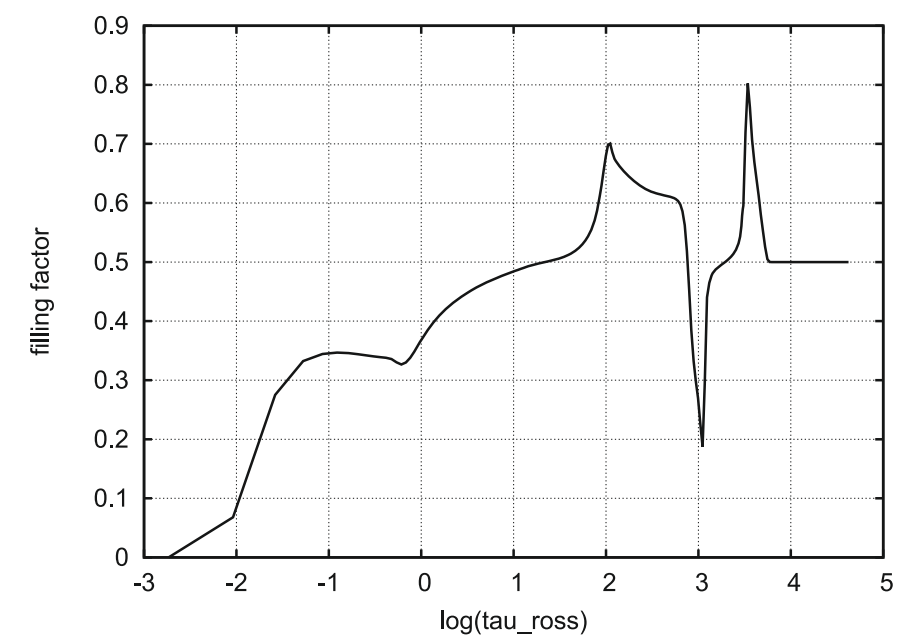

Fig. 2. Filling factor of our model of HD 108642.

we used for this investigation were obtained by Landstreet and Kupka in April 2001 at the CFHT. They observed the star at a very high resolution of $R=120000$ in several chunks of approximately $100 \AA$ in range. We found the ranges 5491-5572 $\AA$ and 6102-6191 A most suitable. These contain enough unblended lines at a reasonable noise level $S / N>140$ and 150 .

As the starting point for our model for the envelope and atmosphere of HD 108642, we took evolutionary envelopes as well as polytropes. We found our code to be insensitive to the differences in these envelope models. Extension into the atmosphere was performed with the gray approximation. For radiation transport we used the OPAL opacities and equation of state (1996) as input data. The only tuneable parameters we have in our model of HD 108642 are its effective temperature of $8100 \mathrm{~K}$, the stars surface gravity of 4.1 and we accounted for its higher metallicity by using opal tables for $Z=0.06$. All other parameters and numerical constants are as described in Kupka and Montgomery (2002). For the line synthesis we adopted the SynthV code of Tsymbal. Its approach of solving the equation of radiative transfer for each atmospheric layer separately naturally allows us to account for depth dependent velocity fields. We performed the radiative transfer twice for each layer, for the upwards and for the downwards moving matter. These two contributions were weighted according to the filling factor and summed. Then, the absorption of the individual layer was added to the total line absorption.

Figure 1 shows the mean velocities of upwards and downwards moving material resulting from our calculations. The values of $\omega_{\mathrm{z}}^{\text {up }}$ and $\omega_{\mathrm{z}}^{\text {down }}$ taken at $\tau_{\text {ross }}=2 / 3$ compare reasonably to the measurement of $v_{\text {mic }}=4.0 \mathrm{~km} \mathrm{~s}^{-1}$ by Landstreet (1998). A comparison of the observed bisector of the CrII line at $4616 \AA$ (Landstreet 1998) and the synthetic bisector derived from the VeDyn model can be seen in Fig. 3. Clearly the magnitude of the calculated bisector is of the same order as the observed one. Also the tilt to the blue is reproduced. However, its shape deviates. This, we believe, is at least in part due to the fact that we could not include the upper part of the atmosphere $\left(\log \left(\tau_{\text {ross }}\right)<-4.0\right)$, because of numerical problems. Furthermore Fig. 1 shows the bisectors of two weak lines, CoI 5342.7 and FeI 5241.9; an encouraging result of our modelling procedure. However, we currently do not have any observations to which to compare those synthetic bisectors. 


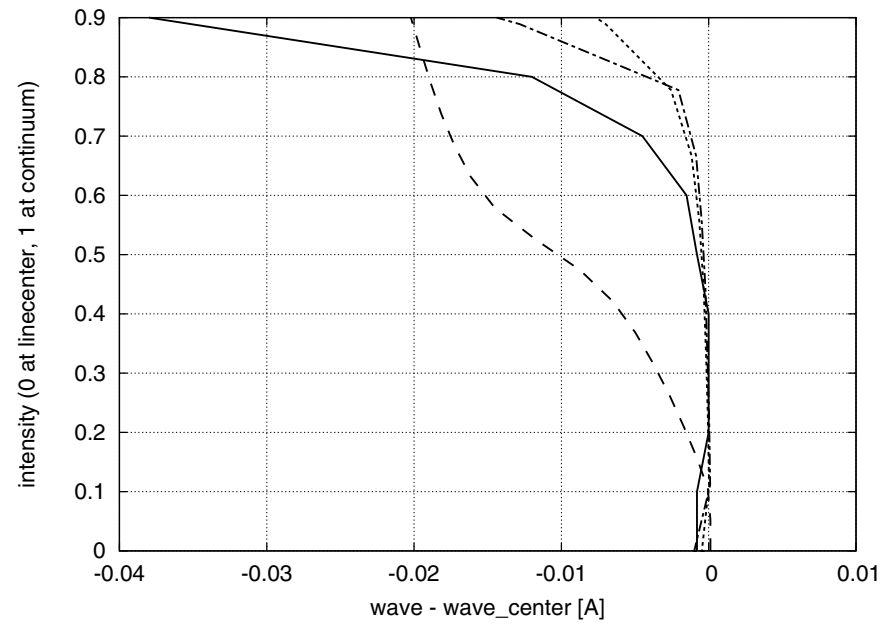

Fig. 3. The solid line shows the observed bisector of CrII 4616 as measured by Landstreet (1998), the dashed line denotes the synthesized bisector. The dotdashed and dotted line represent the calculated bisectors of CoI 5342.7 and FeI 5241.9 respectively; both are weak lines.

\section{Conclusions}

We consistently modeled turbulent convection in the lower atmosphere and envelope of an Am star. Following the model of Canuto and Dubovikov (1998) and the approach of Kupka \& Montgomery (2002), we solved the equations for the turbulent quantities on a weighted mass grid. Other quantities are computed on the same weighted mass grid in the envelope and on a $\log \left(\tau_{\text {ross }}\right)$ grid in the stellar atmosphere. Line synthesis has been performed with a modified version of the SynthV code of Tsymbal, where we account for the depth dependent mean upand downstream velocities and the filling factor.

The resulting line shapes of HD 108642 have been compared to the observed ones. The same has been done for the typical turbulent velocities we derive at optical depths in the vicinity of $\tau_{\text {ross }}=2 / 3$. They are in the range of the microturbulent velocity measured by Landstreet (1998).
We also calculated bisectors from our synthetic line profiles. They are tilted to the blue by a magnitude comparable to the observed ones. However, the shape of the synthetic bisector differs from the observation. We think this results from the upper part of the atmosphere that is missing in our model. Currently more objects are being investigated to cover the region of $\mathrm{A}$ to $\mathrm{F}$ type main sequence stars.

Our modelling and synthesis approach is applicable to investigation methods relying on model atmospheres. The frequency dependent radiation transport still has to be implemented in our models.

Acknowledgements. The author thanks Vadim Tsymbal for letting us alter his synthesis code SynthV, F. Kupka who developed the HME solver and J. Landstreet for the observational data on HD 108642. This research was funded by the FWF project P-18224-N13.

\section{References}

Biermann, L. 1948, ZAp, 25, 135

Canuto, V. M., \& Dubovikov, M. 1998, ApJ, 493, 893

Canuto, V. M., \& Mazzitelli, I. 1991, ApJ, 370, 295

Canuto, V. M., \& Mazzitelli, I. 1992, ApJ, 389, 724

Canuto, V. M., Goldman, I., \& Mazzitelli, I. 1996, ApJ, 473, 550

Canuto, V. M., Cheng, Y., \& Howard, A. 2001, J. Atm. Sci., 58, 1169

Freytag, B. 1995, Ph.D. Thesis, University of Kiel

Freytag, B., \& Steffen, M. 2004, IAUS, 224, 139, The A-star puzzle, ed. J. Zverko, et al. (Cambridge University Press)

Heiter, U., Kupka, F., van't Veer-Menneret, C., et al. 2002, A\&A, 392, 619

Kochukhov, O., Freytag, B., Piskunov, N., et al. 2007, Convection in Astrophysics, Cambridge University Press, ed. F. Kupka, I. Roxburgh, \& K. Chan, IAUS, 239, 68

Kupka, F. 1996, Model Atmospheres and Stellar Spectra, ed. Adelman, et al., ASP Conf. Ser., 108, 73

Kupka, F. 1999, ApJ, 526, L45

Kupka, F., \& Montgomery, M. H. 2002, MNRAS, 330, L6

Kupka, F., \& Muthsam, H. J. 2007, IAUS, 239, 80

Kurucz, R. L. 1993, Kurucz CD-ROM 13, Cambridge, SAO

Landstreet, J. D. 1998, A\&A, 338, 1041

Montgomery, H. M., \& Kupka, F. 2004, MNRAS, 205, 267

Muthsam, H. J., Gob, W., Kupka, F., et al. 1999, NewA, 4, 405

Nordlund, A., \& Stein, R. F. 2000, ASPC, 203, 362

Rogers, F. J., Swenson, F. J., \& Iglesias, C. A. 1996, ApJ, 456, 902

Stein, R. F., \& Nordlund, A. 2003, ASPC, 288, 519 